\title{
30. AfnP-Symposium 2008 Ein Rückblick auf die Tagung in Fulda
}

Das 30. AfnP-Symposium in Fulda war insgesamt ein voller Erfolg. Ein besonderer Dank gilt allen Mitwirkenden und der Industrie für die Unterstützung. Die historische Ausstellung über die Entwicklungen in der Nierenersatztherapie und die Festschrift zum 30-jährigen Jubiläum der AfnP gehörten zu den Highlights auf dem Symposium. Die Vorträge waren in viele Themenbereiche gegliedert und durchweg interessant. Außerdem bot Prof. Gerhard Krönung Shuntworkshops an. Schließlich vergab die AfnP zusammen mit der Dialyse aktuell den diesjährigen Pflegepreis.

Die Vorbereitung und Durchführung des 30. AfnP-Symposiums war in jeder Hinsicht sehr erfolgreich. Der neu gestaltete Stadtsaal, der in sonniger Herbststimmung erstrahlte, bot einen festlichen Rahmen.

\section{Dank an alle Mitwirkenden}

Der Dank gebührt allen Ländervertretungen und Referenten für ihre aktive Mitarbeit, und nicht zuletzt den Förderern seitens der Industrie und der Institutionen. Ohne die Unterstützung der Industrie wäre diese Veranstaltung in den letzten 30 Jahren nicht möglich gewesen. Unser besonderer Dank geht daher an alle Firmen, die in den Jahren immer treu mit ihrer Teilnahme zum Gelingen dieser Veranstaltung beigetragen haben, und das auch in den Zeiten, in denen die Durchführung des AfnPKonzepts schwierig war. Heute präsentiert sich die AfnP in guter Verfassung, was sich im diesjährigen Symposium widerspiegelte.

\section{Ein Blick zurück}

Zum Auftakt des Symposiums begrüßten die AfnP-Vorsitzende Marion Bundschu, Prof. Winfried Fassbinder und der Bürgermeister der Stadt Fulda Herr Dippel die Teilnehmer. Eine Besonderheit für alle Teilnehmer war die Festschrift zum 30. Jubiläum, erstmals mit dem neuen AfnP-Logo. Eindrucksvoll wurden die Historie und die Ziele für die Zukunft der AfnP formuliert. Die historische Ausstellung über den Beginn der Nierenersatztherapie zeigte deutlich die rasante Entwicklung und war für viele Teilnehmer sehr beeindruckend. Hier möchten wir Heidemarie Alexander herzlich für ihr Engagement danken.

\section{Referenten an zwei Tagen}

Mit 64 Referenten, 48 Ausstellern und rund 700 Teilnehmern blicken wir auf ein erfolgreiches Symposium zurück. Das breit gefächerte Themenspektrum spricht für sich: Wer will konnte sich zu den Themen Ernährung und Phos-

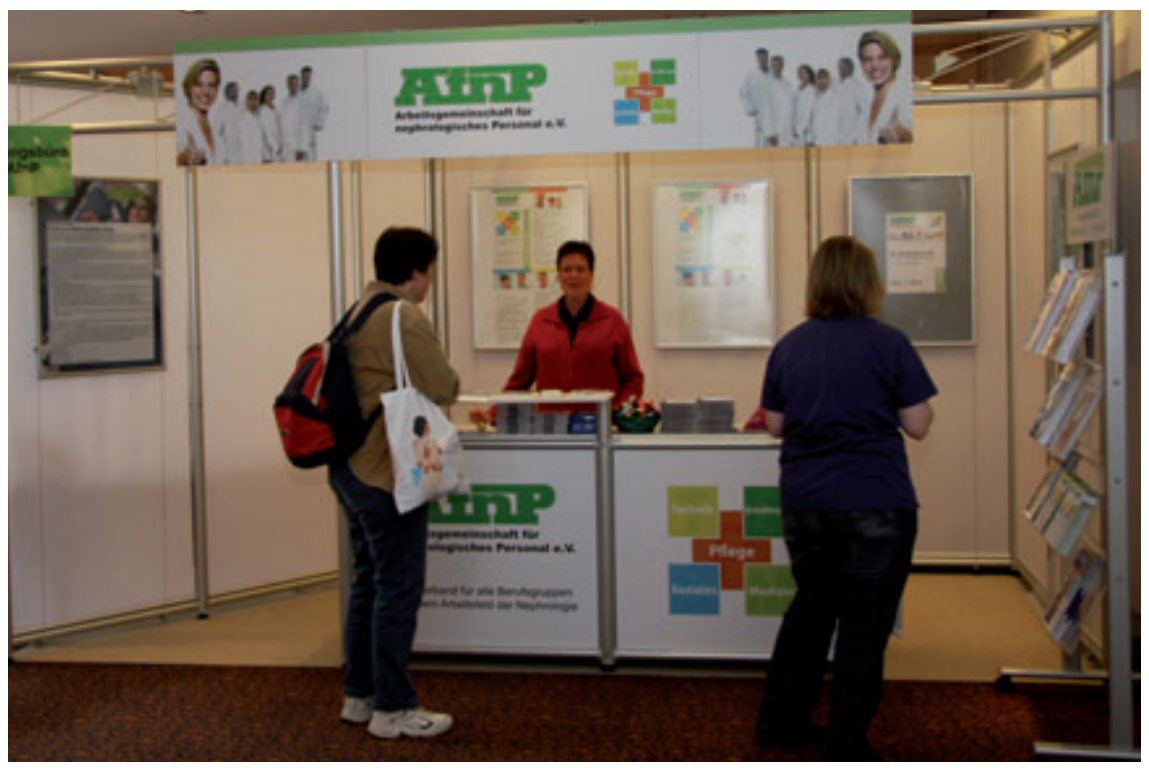

Abb. 1 AfnP-Stand in Fulda. 
phatmanagement, aber auch zu sozialen Themen wie Patientenverfügung und Pflegeversicherung, Aspekten wie Hygiene, Peritonealdialyse, Heimhämodialyse und Notfallmedizin sowie zu technischen Themen fortbilden. Der Vortrag über das aktuelle Problem „Das Pflegeteam wird älter“ war zum 1. Mal zu hören und fand großen Anklang.

Die Arbeitsprozesse in der Pflege sind immer schneller geworden und die Verantwortung ist gestiegen. Spürbar war auch das große Interesse an dem überdurchschnittlich gut besuchten Vortrag von Dr. Felicitas McCarthy zum Thema "Bourn-out”. Unser alljährliches Highlight waren die Themen rund um den Shunt: Ein besonderes Interesse galt hier Prof. Gerhard Krönung in den sehr gut besuchten Workshops. Für uns als Pflegepersonal ist der Shunt des Patienten von großer Bedeutung in der täglichen Dialysepraxis.

\section{Pflegepreis 2008 vergeben}

Der Pflegepreis wurde in diesem Jahr zum 3. Mal von der AfnP und der Dialyse aktuell gemeinsam vergeben. Lesen Sie mehr darüber in diesem Heft.

Wichtige Anregungen von den Teilnehmern wurden aufgegriffen und werden für das kommende Symposium in der Planung berücksichtigt. Wir verabschieden uns mit dem Motto „Man gewinnt immer, wenn man erfährt, was andere von uns denken“" und freuen uns auf ein Wiedersehen 2009.

Elke Fröhlich, Fulda, und Helga Damaschke, Lauterbach

\section{itin}

Arbeitsgemeinschaft für nephrologisches Personal e.V.

So können Sie uns erreichen:

AfnP Geschäftsstelle

Käppelesweg 8

89129 Langenau

Tel.: $07345 / 22933$

Fax: $07345 / 7540$

E-Mail: info@afnp.de

Internet: www.afnp.de

Vorstand der AfnP e.V.

- Marion Bundschu (1. Vorsitzende)

- Hans-Martin Schröder (stellv. Vorsitzender)

- Gabi Steck (Schatzmeisterin)

- Helga Damaschke (Schriftführerin)

\section{Ein Abend mit vielen Erinnerungen ..}

Am Samstag, den 25.10.2008 fand anlässlich des 30. AfnP-Symposiums ein Festabend mit den aktiven Ländervertretern und den Ehren- sowie Gründungsmitgliedern der AfnP statt. Wir können inzwischen auf eine recht erfolgreiche 30-jährige Verbandsgeschichte zurückblicken. Viel ehrenamtlicher Einsatz war schon immer gefragt und heute ist allein die Organisation des jährlichen Symposium mit eine großen Industrieausstellung und Vorträgen in 7 Räumen parallel eine logistische Herausforderung. Besonders haben wir uns über das Kommen der Ehrenmitglieder Richard Beuthin, Jürgen Brunner, Uli Sander, Alice Sücker und Marlene Wolf gefreut. Ohne deren jahrelangen unermüdlichen Einsatz gäbe es die AfnP und auch das jährliche Symposium heute nicht. Unser Dank gilt außerdem den langjährigen, sehr aktiven Ländervertretern Christine Reinheimer-Grüneberg, Diana Wurser, Sigfried Tschöpel, Uwe Stumpf, Marion Frei, Egon Witter, Egon Jans, Margarethe Eilrich und vielen anderen mehr. Eine wichtige Person war all die Jahre Anni Brunner (Ehefrau von Jürgen Brunner), die „Mutter der AfnP“. Ihr wollen wir noch einmal ausdrücklich danken.

Die AfnP e. V. ist heute ein nationaler Berufsverband für alle Berufsgruppen im Arbeitsfeld der Nierenersatztherapie und ihrer verwandten Verfahren geworden. Ihr Image hat sich in den letzen Jahren verändert. Aus einer anfänglich kleinen Gruppe von Gründungsmitgliedern und 100 Mitgliedern ist die AfnP inzwischen auf über 1500 Mitglieder angewachsen. Vor 30 Jahren ahnte noch niemand, wie sich die AfnP einmal entwickeln würde. Ihre Marion Bundschu

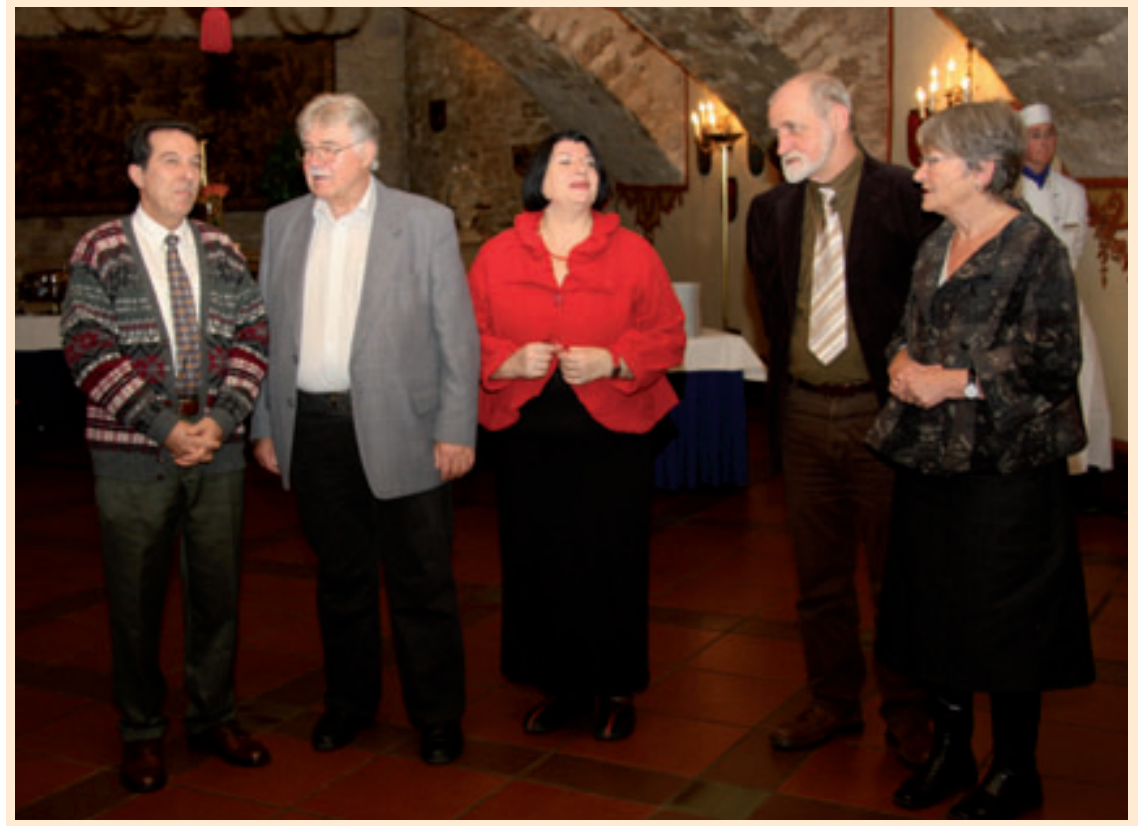

Abb. 2 Unermüdlicher Einsatz für die AfnP: Die Ehrenmitglieder Jürgen Brunner, Richard Beuthin, Marlene Wolf, Uli Sander Alice Sücker (von links).

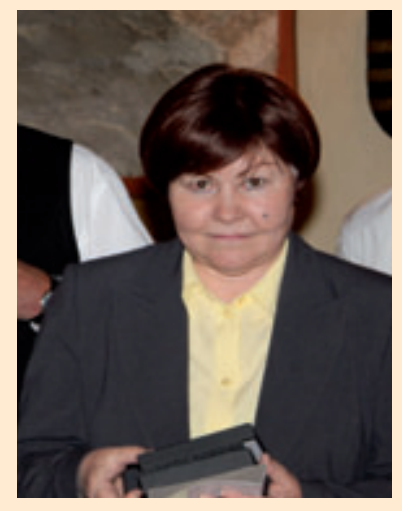

Abb. 3 Heidemarie Alexander ist von Anfang an AfnP-Mitglied und wurde in Fulda für die Organisation der historischen Ausstellung geehrt. 